\title{
Analytical Solutions for Nonlinear Dispersive Physical Model
}

\author{
Wen-Xiu Ma $\mathbb{D}^{1,2,3,4}$ Mohamed R. Ali $\mathbb{D}^{5},{ }^{5}$ and R. Sadat $\mathbb{D}^{6}$ \\ ${ }^{1}$ Department of Mathematics, Zhejiang Normal University, Jinhua 321004, Zhejiang, China \\ ${ }^{2}$ Department of Mathematics, King Abdulaziz University, Jeddah 21589, Saudi Arabia \\ ${ }^{3}$ Department of Mathematics and Statistics, University of South Florida, Tampa, FL 33620-5700, USA \\ ${ }^{4}$ School of Mathematics, South China University of Technology, Guangzhou 510640, China \\ ${ }^{5}$ Department of Basic Science, Faculty of Engineering at Benha, Benha University, 13512, Egypt \\ ${ }^{6}$ Department of Mathematics, Zagazig Faculty of Engineering, Zagazig University, Zagazig, Egypt
}

Correspondence should be addressed to Wen-Xiu Ma; mawx@cas.usf.edu and Mohamed R. Ali; mohamed.reda@bhit.bu.edu.eg

Received 29 May 2020; Accepted 6 July 2020; Published 28 August 2020

Guest Editor: Zhouchao Wei

Copyright (c) 2020 Wen-Xiu Ma et al. This is an open access article distributed under the Creative Commons Attribution License, which permits unrestricted use, distribution, and reproduction in any medium, provided the original work is properly cited.

Nonlinear evolution equations widely describe phenomena in various fields of science, such as plasma, nuclear physics, chemical reactions, optics, shallow water waves, fluid dynamics, signal processing, and image processing. In the present work, the derivation and analysis of Lie symmetries are presented for the time-fractional Benjamin-Bona-Mahony equation (FBBM) with the Riemann-Liouville derivatives. The time FBBM equation is reduced to a nonlinear fractional ordinary differential equation (NLFODE) using its Lie symmetries. These symmetries are derivations using the prolongation theorem. Applying the subequation method, we then use the integrating factor property to solve the NLFODE to obtain a few travelling wave solutions to the time FBBM.

\section{Introduction}

Partial differential equations running into the thinking of most of the researchers as it represented the importance in several topics of scientific fields as mechanics, optical fibers, medical sciences (as breast cancer), biological science, turbulent bursts, and oceans waves [1-11].

The differential model has a broad application in many phenomena as in [12-14]. Recently, nonlinear fractional differential equations (NLFDEs) show significantly in engineering and applications of other sciences, for example, electrochemistry, physics, electromagnetics, and signal data processing [15-22].

Getting exact solutions for these forms of equations became an important issue; then, most researchers try to achieve this target. The most effective method for obtaining exact solutions for NLFPDEs is the Lie symmetry reduction method. There are many papers for using Lie's method to obtain explicit solutions for NLFPDEs [23-26].

In our paper, we drive the symmetry vectors for the time FBBM equation and present new closed-form solutions for it. The FBBM equation has many forms [27-30], and we choose to work on the following form:

$$
D_{t}^{\alpha} \psi=-\psi_{x}-\psi \psi_{x}+\psi_{x x t},
$$

where $\psi_{x x t}$ is the dissipative term.

The manuscript is prearranged as follows. In Section 2, Lie's group method for FPDEs is exposed. In Section 3, we apply the Lie group reduction method to obtain Lie point symmetry for the time FBBM equation (1). At the end of Section 4, we use these similarity variables to get the reduced equation. In Section 4, we use two methods for solving the resulting ordinary differential equation, the first method is the subequation method and the second method is the integrating factors method to get new solutions that have the properties and form the travelling wave form for the FBBME. In the end, conclusions are written in Section 5 .

\section{Notations and Introductory}

2.1. Fractional Riemann-Liouville Derivative. In this section, we show some definitions for RL fractional derivative [31], which can be considered as follows: 


$$
\begin{aligned}
D_{t}^{\alpha} f= \begin{cases}\frac{\mathrm{d}^{n} f(t)}{\mathrm{d} t^{n}}, & \text { if } n=\alpha, n \in \mathbb{N}, \\
\frac{\mathrm{d}^{n}}{\mathrm{~d} t^{n}} I^{n-\alpha} f(t), & \text { if } 0 \leq n-1<\alpha<n, n \in \mathbb{N},\end{cases} \\
I^{n-\alpha} f(t)=\frac{1}{\Gamma(n-\alpha)} \int_{0}^{t}(t-s) f(s) \mathrm{d} s, \quad n>0,
\end{aligned}
$$

where $D_{t}^{\alpha}$ is the total differentiation of integer number of orders $\alpha,(\alpha>0)$, the Gamma function is $\Gamma(n-\alpha)$, and $I^{n} f(t)$ is the (RL) fractional integral of an order of $n$.

Definition 1. The partial derivative of order $\alpha$ for Riemann-Liouville definition is presented by

$$
\partial_{t}^{\alpha}= \begin{cases}\frac{\partial^{n} f}{\partial t^{n}}, & n=\alpha, \\ \frac{\partial^{n}}{\partial t^{n}} \frac{1}{\Gamma(n-\alpha)} \int_{0}^{t}(t-s)^{n-\alpha-1} f(s, x) \mathrm{d} s, & 0 \leq n-1<\alpha<n .\end{cases}
$$

2.2. Notations for Lie Symmetry Reduction Method for the Time FPDEs. In this section, we show in detail the main notations and definitions that will be used for obtaining the symmetries of NLFPDEs. [31-33]

Here, we will consider timing NLFPDEs of the form

$$
\partial_{t}^{\alpha} \psi=F\left(t, x, \psi, \psi_{x}, \psi_{x x}\right), \quad(0<\alpha \leq 1) .
$$

Assume, equation (2) has a Lie vector $\mathrm{X}$ in the form

$$
X=\xi^{1}(x, t, u) \frac{\partial}{\partial x}+\xi^{2}(x, t, u) \frac{\partial}{\partial t}+\eta(x, t, u) \frac{\partial}{\partial u},
$$

where $\xi^{1}, \xi^{2}$, and $\eta$ can be called as the infinitesimals of the transformations the independent and the dependent variables $(x, t, \psi)$, respectively. Let a one-parameter Lie algebra of infinitesimal transformations be of the following form:

$$
\begin{gathered}
\bar{t}=t+\varepsilon \xi^{2}(t, x, \psi)+O\left(\varepsilon^{2}\right), \\
\bar{x}=x+\varepsilon \xi^{1}(t, x, \psi)+O\left(\varepsilon^{2}\right), \\
\bar{\psi}=\psi+\varepsilon \eta(t, x, \psi)+O\left(\varepsilon^{2}\right), \\
\frac{\partial^{\alpha} \bar{\psi}}{\partial \bar{t}}=\frac{\partial^{\alpha} \psi}{\partial t^{\alpha}}+\varepsilon \eta_{\alpha}^{0}(t, x, \psi)+O\left(\varepsilon^{2}\right), \\
\frac{\partial \bar{\psi}}{\partial \bar{x}}=\frac{\partial \psi}{\partial x}+\varepsilon \eta^{x}(t, x, \psi)+O\left(\varepsilon^{2}\right), \\
\frac{\partial^{3} \bar{\psi}}{\partial \bar{x}^{2} \partial \bar{t}}=\frac{\partial^{3} \psi}{\partial x^{2} \partial t}+\varepsilon \eta^{x x t}(t, x, \psi)+O\left(\varepsilon^{2}\right),
\end{gathered}
$$

where $\varepsilon$ " 1 can be defined as a group parameter, in most cases we take it equal one. The explicit expressions of $\eta^{x}, \eta^{x x}$, and $\eta^{x x t}$, which can be called the prolongation of the infinitesimals and are given by

$$
\begin{aligned}
\eta^{x} & =D_{x}(\eta)-\psi_{x} D_{x}\left(\xi^{1}\right)-\psi_{t} D_{t}\left(\xi^{2}\right), \\
\eta^{x x} & =D_{x}\left(\eta^{x}\right)-\psi_{x t} D_{x}\left(\xi^{1}\right)-\psi_{x x} D_{t}\left(\xi^{2}\right), \\
\eta^{x x t} & =D_{x}\left(\eta^{x t}\right)-\psi_{x x x} D_{x}\left(\xi^{1}\right)-\psi_{x x t} D_{x}\left(\xi^{2}\right),
\end{aligned}
$$

where $D_{i}$ is the total differentiation operator [34] with respect to the independent variables $x^{i}(i=1,2$, then $\left.x^{1}=x, x^{2}=t\right)$ :

$$
D_{i}=\frac{\partial}{\partial x^{i}}+\sum_{\alpha=1}^{q} \psi_{i}^{\alpha} \frac{\partial}{\partial \psi^{\alpha}}+\sum_{j=1}^{p} \sum_{\alpha=1}^{q} \psi_{j i}^{\alpha} \frac{\partial}{\partial \psi_{j}^{\alpha}}+\cdots+\sum_{j_{1}=1}^{p} \cdots \sum_{j_{n-1}}^{p} \sum_{\alpha=1}^{q} \psi_{j 1, \ldots, j n-1}^{\alpha} \frac{\partial}{\partial \psi_{1, \ldots, j n-1^{i}}^{\alpha}} .
$$

Theorem 1. Equation (1) concedes a one-parameter group of infinitesimal transformations in equation (2) with the Lie Vector $X$ if and just if the accompanying infinitesimal conditions hold:

$$
\left.\operatorname{Pr}^{(\alpha, 3)} X(\Delta)\right|_{\Delta=0}=0,
$$

where $\Delta=D_{t}^{\alpha} u-F\left(t, x, u, u_{x}, u_{x x}, \ldots ..\right)$ and $\operatorname{Pr}$ is the $3 r d$ prolongation of the infinitesimal generator $X$.

Definition 2. Prolonged vector is given by [31]

$$
\operatorname{Pr}^{(n)} X=X+\sum_{i=1}^{p} \sum_{\alpha=1}^{q} \xi_{i}^{\alpha} \frac{\partial}{\partial u_{i}^{\alpha}}+\cdots+\sum_{j_{1=1}}^{p} \cdots \sum_{j_{n}=1}^{p} \sum_{\alpha=1}^{q} \xi_{j_{1}, \ldots, j_{n}}^{\alpha} \frac{\partial}{\partial u_{j_{1}, \ldots, j_{n}}^{\alpha}},
$$

where $q$ is the numbers of dependent variables, $p$ is the numbers of independent variables, $\partial / \partial u_{j_{1}}^{\alpha}=\partial / \partial u_{x}^{\alpha}$, and PDE involve derivatives up to order $n$. Also, the invariance condition [35] gives

$$
\left.\xi^{2}(t, x, u)\right|_{t=0}=0 .
$$

The $\alpha$ thextended infinitesimal, which deals with fractional derivatives, has the following form [36-38]: 


$$
\eta_{\alpha}^{0}=\frac{\partial^{\alpha} \eta}{\partial t^{\alpha}}+\left(\eta_{u}-\alpha D_{t}\left(\xi^{2}\right)\right) \frac{\partial^{\alpha} u}{\partial t^{\alpha}}-u \frac{\partial^{\alpha} \eta_{u}}{\partial t^{\alpha}}+\mu-\sum_{n=1}^{\infty}\left(\begin{array}{c}
\alpha \\
n
\end{array}\right) D_{t}^{n}\left(\xi^{1}\right) D_{t}^{\alpha-n}\left(u_{x}\right)+\sum_{n=1}^{\infty}\left[\left(\begin{array}{c}
\alpha \\
n
\end{array}\right) \frac{\partial^{n} \eta_{u}}{\partial t^{n}}-\left[\left(\begin{array}{c}
\alpha \\
n+1
\end{array}\right) D_{t}^{n+1}\left(\xi^{2}\right)\right] D_{t}^{\alpha-n}(u)\right]
$$

where

$$
\begin{aligned}
\mu= & \sum_{n=2}^{\infty} \sum_{m=2}^{n} \sum_{k=2}^{m} \sum_{r=2}^{k-1}\left(\begin{array}{c}
\alpha \\
n
\end{array}\right)\left(\begin{array}{l}
n \\
m
\end{array}\right)\left(\begin{array}{l}
k \\
r
\end{array}\right) \frac{1}{k !} \\
& \frac{t^{n-\alpha}}{\Gamma(n+1-\alpha)}[-u]^{r} \frac{\partial^{m}}{\partial t^{m}}\left[u^{k-r}\right] \frac{\partial^{n-m+k}}{\partial t^{n-m} \partial u^{k}} .
\end{aligned}
$$

Remember that

$$
\left(\begin{array}{l}
\alpha \\
n
\end{array}\right)=\frac{(-1)^{n-1} \alpha \Gamma(n-\alpha)}{\Gamma(n-\alpha) \Gamma(n+1)}
$$

Due to linearization of the infinitesimal $\eta$ in $u$ and the presence of $\partial^{k} \eta / \partial \psi^{k}$, $\mu$ will vanish, where $k \geq 2$ in equation (14).

Lemma 1. The function $\psi=\theta(x, t)$ can be defined as an invariant solution of (2) if and only if

$$
\text { (i) } \psi=\theta(x, t) \text { is an invariant surface of equation (2) }
$$$$
\text { (ii) } \xi^{2}(x, t, \theta) \theta_{t}+\xi^{1}(x, t, \theta) \theta_{x}=\eta(x, t, \theta)
$$

\section{Lie Symmetry and Reduction of FBBM Equation}

In this partition, the Lie symmetry reduction method was applied to find the similarity variables for a one-dimensional time (FBBM) equation. Suppose that (1) is an invariant under (2); we have that

$$
\bar{\psi}_{t}^{\alpha}+\overline{\psi \psi}_{x}+\bar{\psi}_{x}-\bar{\psi}_{x x t}=0 .
$$

Thus, $\psi(x, t)$ satisfies equation (1). Applying the third prolongation to (1), we have the accompanying deciding condition, which is given as

$$
\eta_{\alpha}^{0}+\psi_{x} \eta+(\psi+1) \eta^{x}-\eta^{x x t}=0
$$

Substituting (7) and (8) into (16) and equaling coefficients in derivatives for $x$ and power of $u$ to zero, the system of equations is obtained:

$$
\begin{aligned}
& \left(\begin{array}{c}
\alpha \\
n
\end{array}\right) \partial_{t}^{n} \eta_{\psi}-\left(\begin{array}{c}
\alpha \\
n+1
\end{array}\right) D_{t}^{n+1}\left(\xi^{2}\right)=0, \quad n=1,2,3 \ldots \\
& \xi_{\psi}^{2}=\xi_{x}^{2}=\xi_{\psi}^{1}=\xi_{t}^{1}=\eta_{\psi \psi}=0 \\
& (1-\alpha) \xi_{t}^{2}+2 \xi_{x}^{1}=0 \\
& \psi \eta_{x}-\eta_{x x t}+\partial_{t}^{\alpha} \eta-\psi \partial_{t}^{\alpha} \eta_{\psi}+\eta_{x}=0 \\
& 2 \xi_{x t}^{1}-\eta_{t \psi}=0
\end{aligned}
$$

By solving the obtained equations in (18), we get the following infinitesimal:

$$
\begin{aligned}
\xi^{1} & =c_{1}+(\alpha-1) c_{2} x, \\
\xi^{2} & =2 c_{2} t, \\
\eta & =-(\alpha+1) c_{2}(\psi+1),
\end{aligned}
$$

where $c_{1}$ and $c_{2}$ are constants. By the previous infinitesimal, equation (1) has two vector fields in the form

$$
\begin{aligned}
X_{1} & =\frac{\partial}{\partial x}, \\
X_{2} & =(\alpha-1) x \frac{\partial}{\partial x}+2 t \frac{\partial}{\partial t}-(\alpha+1)(\psi+1) \frac{\partial}{\partial \psi} .
\end{aligned}
$$

Case 1. For the infinitesimal generator in (20a), we have a characteristic equation in the following form:

$$
\frac{d x}{1}=\frac{d t}{0}=\frac{d \psi}{0} .
$$

By solving the previous equation, we get the variables $t$ and $\psi$. Putting $\psi=f(t)$ into (1), we obtain the following fractional ODE:

$$
D_{t}^{\alpha} f(t)=0 .
$$

By solving the above equation, we obtain

$$
\psi=a_{1} t^{\alpha-1},
$$

where $a_{1}$ is constant of integration.

Case 2. For $X_{2}$ in equation (20b), the similarity variables for the infinitesimal generator $X_{2}$ can be obtained from the equation:

$$
\frac{\mathrm{d} x}{(\alpha-1) x}=\frac{\mathrm{d} t}{2 t}=-\frac{\mathrm{d} \psi}{(\alpha+1)(\psi+1)} .
$$

The previous equation is called the characteristic equation; by solving it, we have the similarity variable as a result in the form:

$$
\xi=x t^{-((\alpha-1) / 2)} .
$$

The group invariant solution

$$
\psi(x, t)=t^{-((\alpha+1) / 2)} f(\xi)-1=g(\xi)-1,
$$

where $f(\xi)$ is a new arbitrary function of $\xi$ and $g(\xi)=t^{-((\alpha+1) / 2)} f(\xi)$. By using equation (26), equation (1) is transformed into FODE.

Theorem 3. The transformation in (25) and (26), which is obtained from the similarity group method, reduces equation (1) to NLFODE as below: 


$$
\left(P_{2 / \alpha-1}^{(1 / 2)-(3 \alpha / 2), \alpha} f\right)(\xi)-\frac{t^{(\alpha+1) / 2}}{\Gamma(1-\alpha)}+\left(\frac{-3}{2} \alpha+\frac{1}{2}\right) f_{\xi \xi}-\left(\frac{1}{2} \alpha-\frac{1}{2}\right) f_{\xi \xi \xi}=0 .
$$

Using the operator EK fractional differential operator $[32,34]$,

$$
\begin{aligned}
\left(P_{\beta}^{\xi^{2}, \alpha} f\right)(\xi) & =\prod_{j=0}^{n-1}\left(\xi^{2}+j-\frac{1}{\beta} \frac{d}{d \xi}\right)\left(K_{\beta}^{\xi^{2}+\alpha, n-\alpha} f\right)(\xi), \\
n & = \begin{cases}{[\alpha]+1,} & \alpha \neq N, \\
\alpha, & \alpha \in N,\end{cases}
\end{aligned}
$$

where

$$
\left(K_{\beta}^{\xi^{2}, \alpha} f\right)(\xi)= \begin{cases}\frac{1}{\Gamma(\alpha)} \int_{1}^{\infty}(\psi-1)^{\alpha-1} \psi^{-\left(\xi^{2}+\alpha\right)} f\left(\xi \psi^{1 / \beta}\right) d \psi, & \alpha>0 \\ f(\xi), & \alpha=0 .\end{cases}
$$

Proof. Let $n-1<\alpha \leq 1, n=1,2,3,4, \ldots$ Depending on the Riemann-Liouville (RL) derivatives, definitions, and similarity variables in (25) and (26), we obtain

$$
D_{t}^{\alpha} g(\xi)=\frac{\partial^{n}}{\partial t^{n}}\left[\frac{1}{\Gamma(n-\alpha)} \int_{0}^{t}(t-s)^{n-\alpha-1} s^{-((\alpha+1) / 2)} f\left(x s^{((1-\alpha) / 2)}\right) \mathrm{d} s\right] \text {. }
$$

Let $v=t / s$ and $\mathrm{d} s=-\left(t / v^{2}\right) \mathrm{d} v$. Thus, (31) becomes

$$
\begin{aligned}
D_{t}^{\alpha} g(\xi)= & \frac{\partial^{n}}{\partial t^{n}}\left[t^{n-((3 \alpha+1) / 2)} \frac{1}{\Gamma(n-\alpha)} \int_{1}^{\infty}(v-1)^{n-\alpha-1}\right. \\
& \left.\cdot v^{-(n+((-3 \alpha+1) / 2))} f\left(\xi v^{(\alpha-1 / 2)}\right) \mathrm{d} v\right] .
\end{aligned}
$$

Substitute the EK fractional operator in (30) into (32), we have

$$
D_{t}^{\alpha} g(\xi)=\frac{\partial^{n}}{\partial t^{n}}\left[t^{n-((3 \alpha+1) / 2)}\left(K_{(2 / \alpha-1)}^{(1-\alpha) / 2, n-\alpha} f\right)(\xi)\right] .
$$

For simplicity, let $\xi=x t^{-((\alpha-1) / 2)}$ and $\phi \in(0, \infty)$; we acquire.

$$
t(\partial / \partial t) \phi(\xi)=t x(-((\alpha-1) / 2)) t^{-((\alpha-1) / 2)-1} \phi^{\prime}(\xi)=-
$$
$((\alpha-1) / 2) \xi(\partial / \partial \xi) \phi(\xi)$. Hence, equation (33) will be rewritten as

$$
\begin{aligned}
& \frac{\partial^{n}}{\partial t^{n}}\left[t^{n-((3 \alpha+1) / 2)}\left(K_{2 /(\alpha-1)}^{(1-\alpha) / 2, n-\alpha} f\right)(\xi)\right] \\
& =\frac{\partial^{n-1}}{\partial t^{n-1}}\left[\frac{\partial}{\partial t}\left[t^{n-((3 \alpha+1) / 2)}\left(K_{2 /(\alpha-1)}^{(1-\alpha) / 2, n-\alpha} f\right)(\xi)\right]\right] \\
& =\frac{\partial^{n-1}}{\partial t^{n-1}}\left[t^{n-((3 \alpha+1) / 2)-1}\left(n-\left(\frac{3 \alpha+1}{2}\right)-\frac{(\alpha-1)}{2} \xi \frac{\partial}{\partial \xi}\right)\right. \\
& \left.\quad \cdot\left(K_{2 /(\alpha-1)}^{(1-\alpha) / 2, n-\alpha} f\right)(\xi)\right] .
\end{aligned}
$$

Repeating $n-1$ times, we have

$$
\begin{aligned}
& \frac{\partial^{n}}{\partial t^{n}}\left[t^{n-((3 \alpha+1) / 2)}\left(K_{2 /(\alpha-1)}^{(1-\alpha) / 2, n-\alpha} f\right)(\xi)\right] \\
&=\frac{\partial^{n-1}}{\partial t^{n-1}}\left[\frac{\partial}{\partial t}\left[t^{n-((3 \alpha+1) / 2)}\left(K_{2 /(\alpha-1)}^{(1-\alpha) / 2, n-\alpha} f\right)(\xi)\right]\right] \\
&=\frac{\partial^{n-1}}{\partial t^{n-1}}\left[t^{n-((3 \alpha+1) / 2)-1}\left(n-\left(\frac{3 \alpha+1}{2}\right)-\frac{(\alpha-1)}{2} \xi \frac{\partial}{\partial \xi}\right)\right. \\
&\left.\left(K_{2 /(\alpha-1)}^{(1-\alpha) / 2, n-\alpha} f\right)(\xi)\right] \\
& \vdots \\
&= t^{-((3 \alpha+1) / 2)} \prod_{j=0}^{n-1}\left[\left(j-\left(\frac{3 \alpha-1}{2}\right)-\frac{(\alpha-1)}{2} \xi \frac{\partial}{\partial \xi}\right)\right. \\
&\left.\left(K_{2 /(\alpha-1)}^{(1-\alpha) / 2, n-\alpha} f\right)(\xi)\right] .
\end{aligned}
$$

Using the definition of EK fractional differential operator in (28) to rewrite (35), we obtain

$$
D_{t}^{\alpha} g(\xi)=t^{-(3 \alpha+1) / 2}\left(P_{2 /(\alpha-1)}^{(-(3 \alpha+1)) / 2, \alpha} f\right)(\xi) .
$$

Remark 1 . The fractional derivative must achieve the linearization property [37, 39]:

$$
\begin{aligned}
D_{t}^{\alpha}(h(t)+k(t)) & =D_{t}^{\alpha}(h(t))+D_{t}^{\alpha}(k(t)), \\
D_{t}^{\alpha} t^{\gamma} & =\frac{\Gamma(\gamma+1) t^{\gamma-\alpha}}{\Gamma(\gamma-\alpha+1)}, \quad \gamma>\alpha-1 .
\end{aligned}
$$

Using the invariant group solution in (26), (37), and (38), we obtain

$$
D_{t}^{\alpha} \psi(x, t)=D_{t}^{\alpha} g(\xi)-D_{t}^{\alpha}(1) .
$$

Hence,

$$
D_{t}^{\alpha} \psi(x, t)=t^{-(3 \alpha+1) / 2}\left(P_{(2 / \alpha-1)}^{(-(3 \alpha+1)) / 2, \alpha} f\right)(\xi)-\frac{t^{-\alpha}}{\Gamma(1-\alpha)} .
$$

Thus, (1) can be reduced to

$$
\begin{aligned}
& \left(P_{2 /(\alpha-1)}^{(1 / 2)-(3 \alpha / 2), \alpha} f\right)(\xi)-\frac{t^{(\alpha+1) / 2}}{\Gamma(1-\alpha)}+\left(\frac{-3}{2} \alpha+\frac{1}{2}\right) f_{\xi \xi} \\
& -\left(\frac{1}{2} \alpha-\frac{1}{2}\right) \xi f_{\xi \xi \xi}=0,
\end{aligned}
$$

and the theorem is totally proofed.

\section{Explicit Solutions for FBBM Equation}

4.1. Clarifications for the Subequation Method. The subequation method [39] is presented in this section. Consider the NLFPDE in the form 


$$
P\left(\psi, \psi_{t}, \psi_{x}, D_{t}^{\alpha} \psi, D_{x}^{\alpha} \psi, \ldots,\right), \quad(0<\alpha \leq 1),
$$

where $\psi$ is a dependent variable, $P$ is a series of $\psi$ and its fractional derivatives, and $D_{t}^{\alpha} \psi$ and $D_{x}^{\alpha} \psi$ are the Riemann-Liouville (RL) derivatives of $\psi$ w.r.t $t$ and $x$. Here, we present the principles for the subequation technique. By using the d'Alembert transformation,

$$
\psi(x, t)=\psi(\zeta), \quad \zeta=x+c t
$$

where $c$ is constant that will be determined later, and we can rewrite (41) as NLFODE:

$$
P\left(\psi, c \psi^{\prime}, \psi^{\prime \prime}, c^{\alpha} D_{\zeta}^{\alpha} \psi, D_{\zeta}^{\alpha} \psi, \ldots,\right), \quad(0<\alpha \leq 1) .
$$

According to the subequation procedure, assume that the wave solution will be written in the following form:

$$
\psi(\zeta)=a_{0}+\sum_{i-1}^{n} a_{i} \phi(\zeta)^{i}
$$

where $a_{i},(i=1, \ldots, n)$ are constants, which will be determined later, $n$ belongs to integers numbers, which are determined by equaling the highest order derivatives and nonlinear terms in (44) together, and the function $\phi(\zeta)$ achieves the Riccati equation of fractional order

$$
D_{\zeta}^{\alpha} \phi(\zeta)=\sigma+\phi(\zeta)^{2}
$$

where $\sigma$ is a constant. Some trigonometric solutions of the fractional Riccati equation (46) are

$$
\phi(\zeta)= \begin{cases}-\sqrt{-\sigma} \tan h_{\alpha}(\sqrt{-\sigma} \zeta), & \sigma<0, \\ -\sqrt{-\sigma} \cot h_{\alpha}(\sqrt{-\sigma} \zeta), & \sigma<0, \\ \sqrt{\sigma} \tan _{\alpha}(\sqrt{\sigma} \zeta), & \sigma>0, \\ -\sqrt{\sigma} \cot _{\alpha}(\sqrt{\sigma} \zeta), & w>0, \\ \frac{-\Gamma(1+\alpha)}{\zeta^{\alpha}+w}, & w=\text { constant, } \sigma=0\end{cases}
$$

By substituting forms (45) into (44) and setting the coefficients of $\phi(\zeta)$ to be zero, we obtain an algebraic system in $a_{i},(i=1, \ldots, n)$ and $c$. By solving the determinate system, we obtain the constants $a_{i},(i=1, \ldots, n)$ and $c$. Substituting these constants and the solutions of (47) into (45), we obtain the closed form solutions of (42).

4.2. Applying the Subequation Method to the Time FBBM Equation. We now implement a subequation method to (1). We will use the transformation

$$
\psi(x, t)=\psi(\zeta), \quad \zeta=x+c t
$$

where $c$ is a constant, and this will transform (1) into an NLFODE:

$$
c^{\alpha} D_{\zeta}^{\alpha} \psi=-\psi_{\zeta}-\psi \psi_{\zeta}+C \psi_{\zeta \zeta \zeta}
$$

We now assume that (49) has the solution in the form

$$
\psi(\zeta)=a_{0}+\sum_{i-1}^{n} a_{i} \phi(\zeta)^{i},
$$

where $a_{i}(i=1, \ldots, n)$ are constants, which will be determined, and $\phi(\zeta)$ achieves equation (46).

Balancing the highest order derivative terms with nonlinear terms in equation (49), we obtain $n=2$. Hence,

$$
\psi(\zeta)=a_{0}+a_{1} \phi(\zeta)+a_{2} \phi(\zeta)^{2} .
$$

We then substitute (51) along with (46) into (49), then collect the coefficients of $\phi(\zeta)$, and set them to equal zero. A set of algebraic equations are obtained in knowns $c$, $a_{0}, a_{1}$, and $a_{2}$. Solving these algebraic equations with the help of the software program (Maple), we get the following values.

Thus, from (47), we obtain five forms of explicit travelling wave solutions of (1), namely,

$$
\begin{aligned}
c & =\frac{1}{12} \sigma, \\
\sigma & =\sigma, \\
a_{0} & =-c^{\alpha}-1+\frac{2}{3} \sigma^{2}, \\
a_{1} & =0, \\
a_{2} & =\sigma .
\end{aligned}
$$

Thus, from (47), we obtain five forms of explicit travelling wave solutions of (1), namely,

$$
\begin{aligned}
& \psi_{1}(x, t)=a_{0}+\sigma\left(-\sqrt{-\sigma} \tan h_{\alpha}(\sqrt{-\sigma}(x+c t))\right)^{2}, \quad \sigma<0, \\
& \psi_{2}(x, t)=a_{0}+\sigma\left(-\sqrt{-\sigma} \cot h_{\alpha}(\sqrt{-\sigma}(x+c t))\right)^{2}, \quad \sigma<0 \\
& \psi_{3}(x, t)=a_{0}+\sigma\left(\sqrt{\sigma} \tan _{\alpha}(\sqrt{\sigma}(x+c t))\right)^{2}, \quad \sigma>0 \\
& \psi_{4}(x, t)=a_{0}+\sigma\left(-\sqrt{\sigma} \cot _{\alpha}(\sqrt{\sigma}(x+c t))\right)^{2}, \quad \sigma>0 \\
& \psi_{5}(x, t)=a_{0}+\sigma\left(\frac{-\Gamma(1+\alpha)}{(x+c t)^{\alpha}+w}\right)^{2}, \quad \sigma=0
\end{aligned}
$$

where $a_{0}$ is arbitrary constant. We plot the result in equation (57) in the three dimensions, contour plot, and density plot, as shown in Figures 1-3, respectively.

4.3. Applying Simple Transformation. We solve the conformable FBBM equation using simple transformation to change the fraction order in partial derivative to nonsolvable ODE. For the reduction of (1) to ODE, we use the following transformation: 


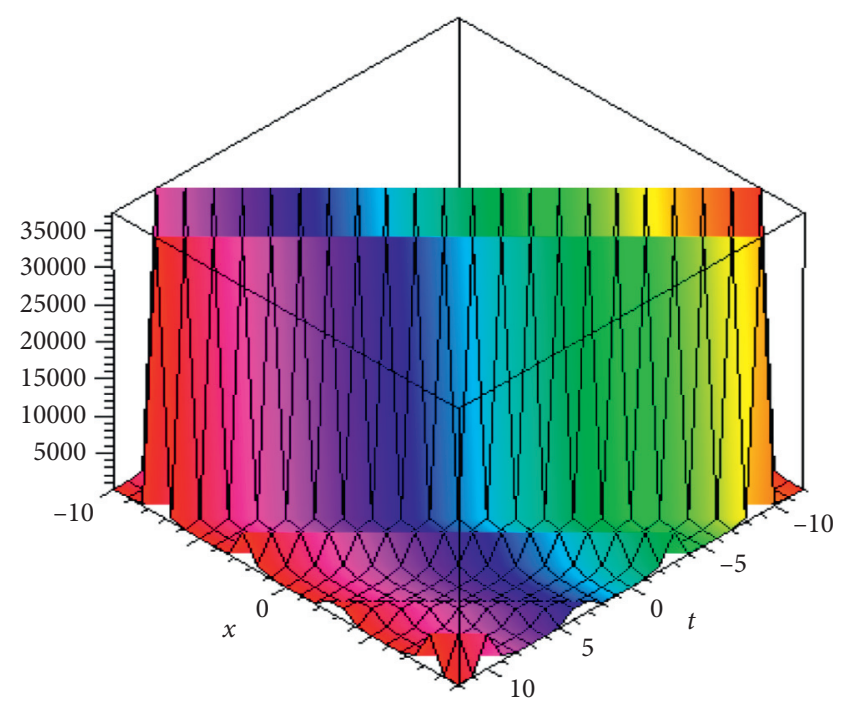

FIgURE 1: 3D plot for (57) with $\sigma=10$ and $\alpha=0.75$.

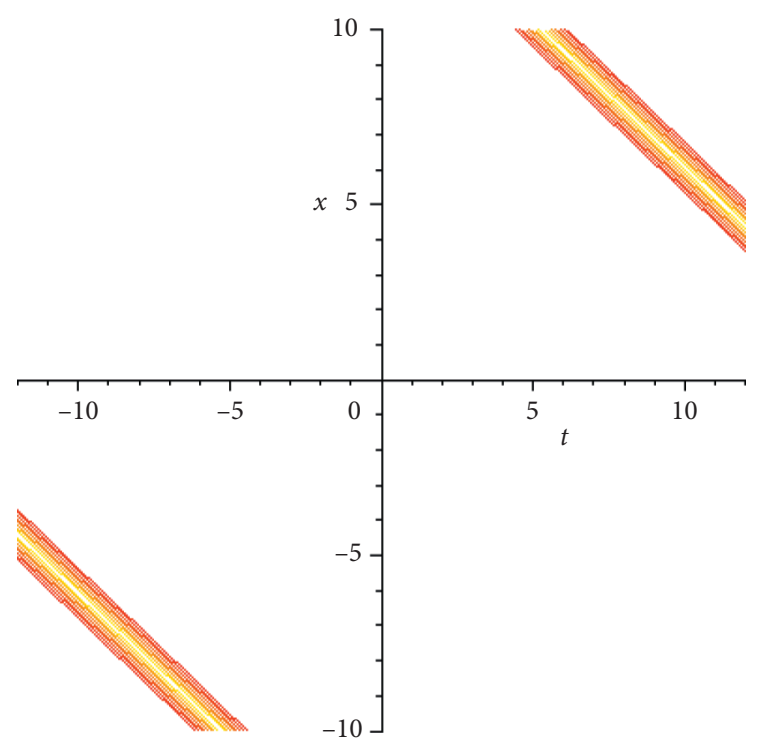

Figure 2: Contour plot of (57) at $\sigma=10$ and $\alpha=0.75$.

$$
\psi(x, t)=\psi(\zeta), \quad \text { where } \zeta=v x-k \frac{t^{\alpha}}{\alpha}
$$

where $v$ and $k$ are arbitrary constants; we can rewrite (1) as NLODE:

$$
k v^{2} \psi_{\zeta \zeta \zeta}=(k-v) \psi_{\zeta}-v \psi \psi_{\zeta}
$$

This equation has no implicit solution but possesses two integrating factors. We apply the integrating factor technique to obtain an analytical solution for (59).

Equation (59) has two integrating factors (IF) as follows:

$$
\begin{aligned}
& \mu_{1}=\psi(\zeta), \\
& \mu_{2}=1 .
\end{aligned}
$$

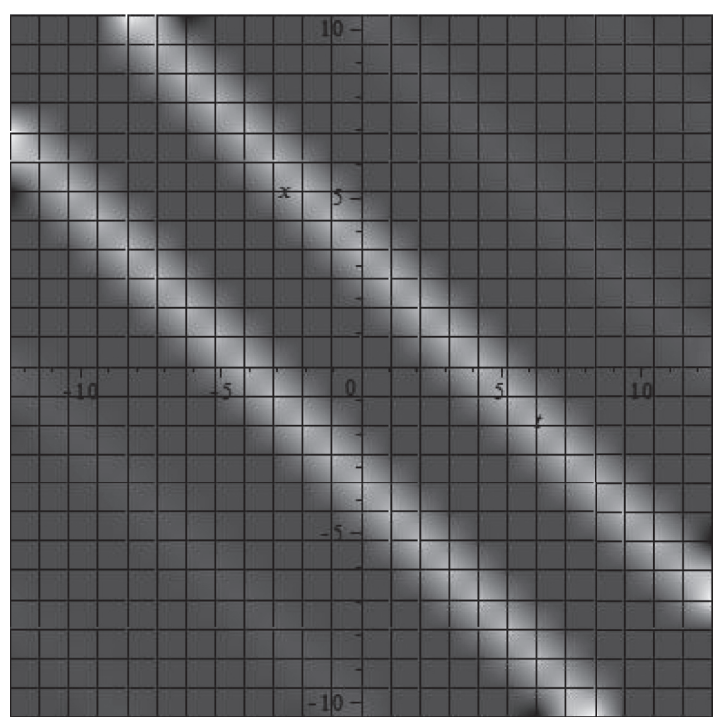

Figure 3: Density plot of (57) at $\sigma=10$ and $\alpha=0.75$.

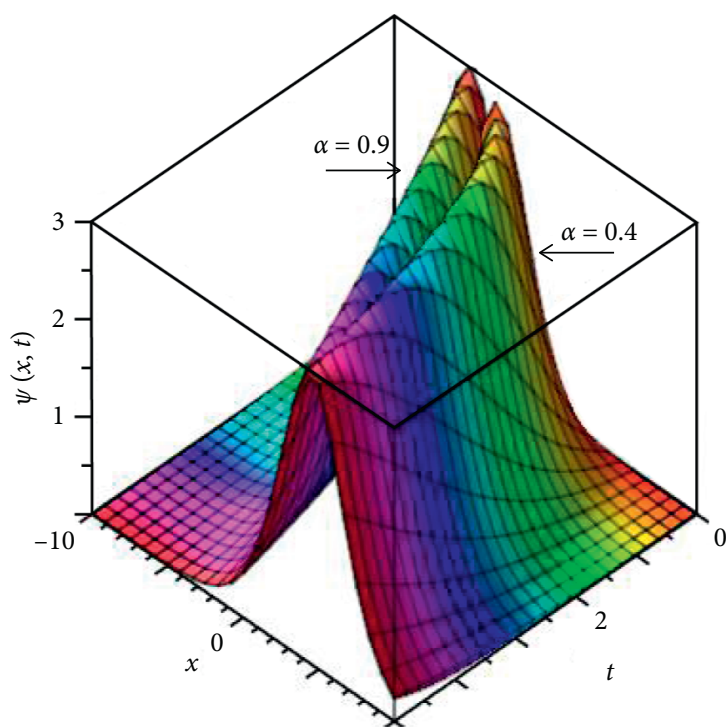

Figure 4: $\psi(x, t)$ at $c=1, v=-1, k=1$, and $\alpha=0.4,0.9$.

Using these integrating factors by the same steps in [39] and neglecting the constants of integration, equation (59) will be reduced to

$$
3 k v^{2}\left(\psi_{\zeta}\right)^{2}=3(k-v) \psi^{2}-v \psi^{3}
$$

By solving this equation, we obtain travelling wave solution for (1):

$$
\begin{aligned}
\psi(\zeta)= & \frac{1}{v}\left(3 \left(-v \tan \left(\frac{1}{2} \frac{\sqrt{-k^{2}+k v}(-\zeta+c)}{k v}\right)^{2}\right.\right. \\
& \left.\left.+k-v+k \tan \left(\frac{1}{2} \frac{\sqrt{-k^{2}+k v}(-\zeta+c)}{k v}\right)^{2}\right)\right) .
\end{aligned}
$$




$$
\begin{aligned}
\text { Replacing } \zeta=v x-k\left(t^{\alpha} / \alpha\right) \\
\psi(x, t)=\frac{1}{v}\left(3\left(-v \tan \left(\frac{1}{2} \frac{\sqrt{-k^{2}+k v}\left(-v x+k\left(t^{\alpha} / \alpha\right)+c\right)}{k v}\right)\right)^{2}\right. \\
\left.+k-v+k \tan \left(\frac{1}{2} \frac{\sqrt{-k^{2}+k v}\left(-v x+k\left(t^{\alpha} / \alpha\right)+c\right)}{k v}\right)^{2}\right) .
\end{aligned}
$$

In other manner, equation (59) have two Lie vectors. The first one of them reduces it to

$$
f_{r r}=\frac{-\left(f_{r}\right)^{2}}{f}-\frac{-k+v+k r}{k v^{2} f}, \quad \text { where, } f=\psi_{\zeta}, r=\psi \text {. }
$$

Equation (64) has closed form solution, but, in the back substitution step, we are unable to get $\psi(x, t)$ even if we neglect the values of constants. So, from here, we can say the integrating factor method for reducing and solve ODEs, occasionally, more effectiveness than the Lie reduction method. Result obtained in (63) is plotted in Figure 4 at different values of $\alpha$. We observe that, by decreasing the value of $\alpha$, the top of the wave has a parabolic shape.

Comparing our result in (63) with results in [5], specially equation (17), we find that the two solutions are travelling wave solutions, but the amplitude and direction of flow are different.

\section{Conclusions}

In this paper, we show the importance and the effective of the Lie symmetry reduction method on the FBBM equations. We obtain time FBBM equation's Lie symmetry generators and then reduce the equation to FODE using these symmetry vectors. The projected analysis is extremely effective and dependable for getting similarity solutions for fractional differential equations. New travelling solutions were derived for the FBBM equation using the subequation method.

\section{Data Availability}

The data used to support the findings of this study are available from the corresponding author upon request.

\section{Conflicts of Interest}

The authors declare that they have no conflicts of interest.

\section{References}

[1] N. Savaissou, B. Gambo, H. Rezazadeh, A. Bekir, and S. Y. Doka, "Exact optical solitons to the perturbed nonlinear Schrödinger equation with dual-power law of nonlinearity," Optical and Quantum Electronics, vol. 52, no. 6, p. 318, 2020.

[2] W. Gao, H. Rezazadeh, Z. Pinar, H. M. Baskonus, S. Sarwar, and G. Yel, "Novel explicit solutions for the nonlinear Zoomeron equation by using newly extended direct algebraic technique," Optical and Quantum Electronics, vol. 52, no. 1, p. 52, 2020.

[3] N. Raza, U. Afzal, A. R. Butt, and H. Rezazadeh, "Optical solitons in nematic liquid crystals with Kerr and parabolic law nonlinearities," Optical and Quantum Electronics, vol. 51, no. 4, p. 107, 2019.

[4] H. Rezazadeh, A. Korkmaz, M. Eslami, and S. M. MirhosseiniAlizamini, "A large family of optical solutions to Kundu-Eckhaus model by a new auxiliary equation method," Optical and Quantum Electronics, vol. 51, no. 3, p. 84, 2019.

[5] Korkmaz, A., Exact solutions to some conformable time fractional equations in Benjamin-Bona-Mahony family, 2016.

[6] M. R. Ali and W.-X. Ma, "New exact solutions of Bratu Gelfand model in two dimensions using Lie symmetry analysis," Chinese Journal of Physics, vol. 65, pp. 198-206, 2020.

[7] M. R. Ali and W.-X. Ma, "New exact solutions of nonlinear $(3+1)$-dimensional boiti-leon-manna-pempinelli equation," Advances in Mathematical Physics, vol. 2019, no. 34, 7 pages, 2019.

[8] M. R. Ali and D. Baleanu, "Haar wavelets scheme for solving the unsteady gas flow in four-dimensional," Thermal Science, vol. 24, no. 2 Part B, pp. 1357-1367, 2019.

[9] M. R. Ali, "A truncation method for solving the time-fractional benjamin-ono equation," Journal of Applied Mathematics, vol. 2019, no. 18, 7 pages, 2019.

[10] M. R. Ali and A. R. Hadhood, "Hybrid orthonormal bernstein and block-pulse functions wavelet scheme for solving the $2 \mathrm{D}$ Bratu problem," Results in Physics, vol. 12, pp. 525-530, 2019.

[11] R. Saleh, R. Sadat, and M. Kassem, "Optimal solutions of a $(3+1)$-dimensional B-Kadomtsev-Petviashvii equation," Mathematical Methods in the Applied Sciences, vol. 43, no. 4, pp. 1775-1787, 2020.

[12] Z. Sabir, H. A. Wahab, M. Umar, M. G. Sakar, and M. A. Z. Raja, "Novel design of Morlet wavelet neural network for solving second order Lane-Emden equation," Mathematics and Computers in Simulation, vol. 172, pp. 1-14, 2020.

[13] Z. Sabir, H. A. Wahab, M. Umar, and F. Erdoğan, "Stochastic numerical approach for solving second order nonlinear singular functional differential equation," Applied Mathematics and Computation, vol. 363, Article ID 124605, 2019.

[14] Z. Sabir, F. Amin, D. Pohl, and J. L. G. Guirao, "Intelligence computing approach for solving second order system of Emden-Fowler model," Journal of Intelligent \& Fuzzy Systems, vol. 38, no. 6, pp. 7391-7406, 2020.

[15] B. Ahmad, S. K. Ntouyas, and A. Alsaedi, "On a coupled system of fractional differential equations with coupled nonlocal and integral boundary conditions," Chaos, Solitons \& Fractals, vol. 83, pp. 234-241, 2016.

[16] A. I. Aliyu and M. Al-Qurashi, "Invariant subspace and approximate analytic solutions of a fractional model of convective longitudinal fins in thermal conductivity," The European Physical Journal Plus, vol. 134, no. 9, p. 417, 2019.

[17] T. F. Nonnenmacher and R. Metzler, "Nonnenmacher and ralf metzler, on the riemann-liouville fractional calculus and some recent applications," Fractals, vol. 3, no. 3, pp. 557-566, 1995.

[18] A. I. Aliyu, A. S. Alshomrani, Y. Li, M. Inc, and D. Baleanu, "Existence theory and numerical simulation of HIV-I cure model with new fractional derivative possessing a non-singular kernel," Advances in Difference Equations, vol. 2019, no. 1, p. 408, 2019.

[19] B. Lu, "Bäcklund transformation of fractional Riccati equation and its applications to nonlinear fractional partial differential equations," Physics Letters A, vol. 376, no. 28-29, pp. 2045-2048, 2012.

[20] A. Isa Aliyu, Y. Li, M. Inc, and D. Baleanu, "Invariant subspaces, exact solutions and classification of conservation laws for a coupled $(1+1)$-dimensional nonlinear Wu-Zhang equation," Physica Scripta, vol. 95, no. 3, 2020. 
[21] K. Oldham and J. Spanier, The Fractional Calculus Theory and Applications of Differentiation and Integration to Arbitrary Order, Elsevier, Amsterdam, Netherlands, 1974.

[22] K. Shah, H. Khalil, and R. A. Khan, "Investigation of positive solution to a coupled system of impulsive boundary value problems for nonlinear fractional order differential equations," Chaos, Solitons \& Fractals, vol. 77, pp. 240-246, 2015.

[23] D. Baleanu, M. Inc, A. Yusuf et al., "Lie symmetry analysis, exact solutions and conservation laws for the time fractional modified Zakharov-Kuznetsov equation," Nonlinear Analysis: Modelling and Control, vol. 22, no. 6, pp. 861-876, 2017.

[24] D. Baleanu, M. Inc, A. Yusuf, and A. I. Aliyu, "Lie symmetry analysis, exact solutions and conservation laws for the time fractional Caudrey-Dodd-Gibbon-Sawada-Kotera equation," Communications in Nonlinear Science and Numerical Simulation, vol. 59, pp. 222-234, 2018.

[25] G.-W. Wang, X.-Q. Liu, and Y.-Y. Zhang, "Lie symmetry analysis to the time fractional generalized fifth-order $\mathrm{KdV}$ equation," Communications in Nonlinear Science and $\mathrm{Nu}$ merical Simulation, vol. 18, no. 9, pp. 2321-2326, 2013.

[26] M. Inc, A. Yusuf, A. I. Aliyu, and D. Baleanu, "Time-fractional Cahn-Allen and time-fractional Klein-Gordon equations: lie symmetry analysis, explicit solutions and convergence analysis," Physica A: Statistical Mechanics and Its Applications, vol. 493, pp. 94-106, 2018.

[27] Q. Feng and F. Meng, "Traveling wave solutions for fractional partial differential equations arising in mathematical physics by an improved fractional Jacobi elliptic equation method," Mathematical Methods in the Applied Sciences, vol. 40, no. 10, pp. 3676-3686, 2017.

[28] O. Kolebaje and O. Popoola, "Assessment of the exact solutions of the space and time fractional benjamin-bonamahony equation via the-expansion method, modified simple equation method, and liu's theorem," ISRN Mathematical Physics, vol. 2014, Article ID 217184, 11 pages, 2014.

[29] J. A. Pava, "Stability properties of solitary waves for fractional $\mathrm{KdV}$ and BBM equations," Nonlinearity, vol. 31, no. 3, pp. 920-956, 2018.

[30] B. Zheng, "A new fractional Jacobi elliptic equation method for solving fractional partial differential equations," Advances in Difference Equations, vol. 2014, no. 1, p. 228, 2014.

[31] S. S. Ray and S. Sahoo, "Invariant analysis and conservation laws of $(2+1)$ dimensional time-fractional ZK-BBM equation in gravity water waves," Computers \& Mathematics with Applications, vol. 75, no. 7, pp. 2271-2279, 2018.

[32] G.-W. Wang and T.-Z. Xu, "Invariant analysis and exact solutions of nonlinear time fractional Sharma-Tasso-Olver equation by Lie group analysis," Nonlinear Dynamics, vol. 76, no. 1, pp. 571-580, 2014.

[33] R. Gazizov, A. Kasatkin, and S. Y. Lukashchuk, "Continuous transformation groups of fractional differential equations," Vestnik Usatu, vol. 9, no. 3, p. 21, 2007.

[34] P. J. Olver, Applications of Lie Groups to Differential Equations, Springer Science \& Business Media, Berlin, Germany, 2000.

[35] G. F. Jefferson and J. Carminati, "FracSym: automated symbolic computation of Lie symmetries of fractional differential equations," Computer Physics Communications, vol. 185, no. 1 , pp. 430-441, 2014.

[36] R. Khalil, M. Al Horani, A. Yousef, and M. Sababheh, "A new definition of fractional derivative," Journal of Computational and Applied Mathematics, vol. 264, pp. 65-70, 2014.

[37] V. S. Kiryakova, Generalized Fractional Calculus and Applications, CRC Press, Boca Raton, FL, USA, 1993.
[38] M. Eslami and H. Rezazadeh, "The first integral method for Wu-Zhang system with conformable time-fractional derivative," Calcolo, vol. 53, no. 3, pp. 475-485, 2016.

[39] S. Zhang and H.-Q. Zhang, "Fractional sub-equation method and its applications to nonlinear fractional PDEs," Physics Letters A, vol. 375, no. 7, pp. 1069-1073, 2011. 